\title{
Original
}

\section{Osteogenic Potential of Human Dental Follicle Cells on Rat Calvaria}

\author{
Satoshi Iwai' ${ }^{1)}$ Kayo Kuyama ${ }^{2,4)}$ Noboru Kuboyama $^{3,4)}$, Shimpei Takiguchi, 4), \\ Naomi Ogura ${ }^{1,4)}$, Hirotsugu Yamamoto ${ }^{2,4)}$ and Toshirou Kondoh ${ }^{1,4)}$ \\ ${ }^{1)}$ Departments of Maxillofacial Surgery, Nihon University School of Dentistry at Matsudo, Chiba, Japan \\ ${ }^{2)}$ Department of Oral Pathology, Nihon University School of Dentistry at Matsudo, Chiba, Japan \\ ${ }^{3}$ Department of Pharmacology, Nihon University School of Dentistry at Matsudo, Chiba, Japan \\ ${ }^{4)}$ Research Institute of Oral Science, Nihon University School of Dentistry at Matsudo, Chiba, Japan \\ (Accepted for publication, November 20,2012)
}

\begin{abstract}
The objective of this study was to investigate the osteogenic potential of human dental follicle cells (hDFC) in vivo. hDFC were isolated from dental follicle tissue by enzymatic digestion and cultured in growth medium (GM). hDFC were grown in a three-dimensional (3D) culture using gelatin sponges in osteogenic induction medium (OIM) or GM. The cells were transplanted onto calvaria bone of immunodeficient rats $(\mathrm{n}=$ 3). Hematoxylin and eosin (H-E) staining and immunohistochemistry were performed, and bone formation was analyzed with micro-computed tomography (micro-CT). H-E staining showed newly formed bone after transplantation of hDFC grown in a 3D culture in OIM. Immunohistochemistry showed BMP-2, Runx2, and Osterix expression in the hDFC transplantation site during the early stage of bone formation. Moreover, microCT showed that the transplanted hDFC that were 3D-cultured in OIM promoted good bone quality and bone volume compared to the other two groups. Thus, human dental follicles may be a potentially useful cell source for regenerative therapy.
\end{abstract}

Key words: Dental follicle cells, Osteogenic potential, Transplantation, Three-dimensional culture, Microcomputed tomography

\section{Introduction}

The therapeutic approaches for tissue-engineered repair of bone defects have so far concentrated on osteoblasts, lineagecommitted stem/progenitor cells, appropriate extracellular matrix scaffolds, and the signaling pathways important for bone tissue engineering. Mesenchymal stem cells (MSC) from bone marrow can be isolated and expanded through several passages while retaining their multipotent differentiation capacity ${ }^{1,2)}$. However, bone marrow harvesting is invasive, and many alternative sources of MSC are also considered to be potentially useful in tissue engineering, including adipose tissue ${ }^{3,4)}$ and dental pulp ${ }^{5,6}$.

Considerable attention has been paid to human dental follicle cells (hDFC) that originate in the dental follicle, which is a loose ectomesenchymally derived connective tissue surrounding the enamel organ and the dental papilla of the developing tooth germ prior to eruption ${ }^{7)}$. This tissue contains stem cells and lineagecommitted progenitor cells or precursor cells of osteoblasts/ cementoblasts. hDFC also have the capacity to form various types of cells, not only of the osteoblastic/cementoblastic lineage ${ }^{1,8)}$, Corresponding author: Dr. Toshirou Kondoh, Departments of Maxillofacial Surgery, Nihon University School of Dentistry at Matsudo, 2-870-1 Sakaecho-Nishi, Matsudo, Chiba, 271-8587, Japan .Tel/Fax: +81 47360 9394, E-mail: kondo.toshiro@nihon-u.ac.jp but also of the adipogenic and neurogenic lineages ${ }^{2,9)}$, when they receive appropriate signals. hDFC can be very easily obtained because they are exposed in several surgical operations such as extraction of impacted teeth. Controlling differentiation of hDFC into osteoblasts could produce a useful source of bone progenitors for regeneration. The characteristics of hDFC have been compared to those of bone marrow MSC, and their gene expression was profiled during osteogenic differentiation in vitro ${ }^{10,11)}$. These studies indicated that the expression of cell markers and growth factor receptors by hDFC and hMSC was similar.

In the present study, we observed the effect of the osteogenic potential in hDFC in vivo. hDFC were cultured in a 3-dimensional (3D) scaffold in growth medium (GM) or osteogenic induction medium (OIM) and transplanted into the area between the periosteum and the calvarial bone of immunodeficient rats using a polytetrafluoroethylene (PTFE) tube. The osteogenic potential of hDFC 3D culture was evaluated by histology and microcomputed tomography (micro-CT).

\section{Materials and Methods}

\section{Cell culture}

hDFC were obtained using a previously reported method ${ }^{1)}$. 
hDFC were obtained from patients who gave complete informed consent. Normal human impacted third molars were surgically removed and collected. Dental follicle tissues were digested in a solution of $0.1 \mathrm{U} / \mathrm{ml}$ collagenase type $\mathrm{I}$ and $1 \mathrm{U} / \mathrm{ml}$ dispase (Roche, Mannheim, Germany) for $1 \mathrm{~h}$ at $37^{\circ} \mathrm{C}$. The attached hDFC were cultured in 100-mm dishes using MSC growth medium (GM; MSC basal medium plus SingleQuonts ${ }^{\mathrm{TM}}$ growth supplements, which contained fetal bovine serum (FBS), L-glutamine, and penicillin/ streptomycin; Lonza Walkersville, Walkersville, MD, USA) in a humidified incubator $\left(\mathrm{CO}_{2}\right.$ incubator MCO-175M; Sanyo Electric Medical System, Osaka, Japan) in the presence of $95 \%$ air and $5 \% \mathrm{CO}_{2}$ at $37^{\circ} \mathrm{C}$. The medium was changed twice a week. hDFC experiments were performed according to the guidelines established by the Institutional Review Board of Nihon University School of Dentistry at Matsudo (Ethics Committee Registration No: 05-025).

\section{Three-dimensional culture}

MedGel Scaffold ( $\beta$-tricalcium phosphate $(\beta$-TCP) strengthening-type gelatin sponge (MedGel, Kyoto, Japan)) was used for 3D culture. The scaffold $[3 \mathrm{~mm}$ (diameter) $\times 2 \mathrm{~mm}$ (height), pore size: $100-300 \mu \mathrm{m}]$ was washed with PBS and cell culture medium before use. hDFC were suspended in GM at $5.0 \times$ $10^{5} \mathrm{cells} / \mathrm{ml}$, and then $\mathrm{hDFC}$ suspension $(25 \mu \mathrm{l})$ was placed on the scaffold in 96-well plates. GM or MSC osteogenic induction medium (OIM; MSC basal medium and osteogenic SingleQuonts ${ }^{\mathrm{TM}}$ that contained FBS, L-glutamine, penicillin/ streptomycin, dexamethasone, ascorbate, and $\beta$-glycerophosphate; Lonza Walkersville) was added after $1 \mathrm{~h}$, and the cells were cultured in $\mathrm{CO}_{2}$ incubator for the indicated times.

\section{Scanning electron microscopy (SEM)}

After hDFC 3D culture, each hDFC sample was observed by SEM (S-2700, Hitachi, Japan) at numerous magnifications at 15 $\mathrm{kV}$. The hDFC 3D culture was fixed in $10 \%$ neutral-buffered formalin solution for $1 \mathrm{~h}$ at room temperature, washed three times with PBS, dehydrated with 50,60, 70, 80, 90, and 100-vol\% ethanol aqueous solutions, immersed in tertiary-butyl alcohol, and then freeze-dried under a vacuum (FDU-1200, EYELA, Japan). Finally, samples were mounted onto aluminum stubs and sputtercoated with a platinum-palladium alloy before imaging.

\section{Transplantation procedure}

The transplantation procedure was designed to evaluate bone formation of hDFC in vivo (Fig. 1A). F344/NJcl-rnu/rnu male rats (immunodeficient rats, 9 weeks old, weighing 150-200 g, total of 18 rats) were purchased from Japan Crea (Tokyo, Japan). The rats were randomly divided into six groups ( $\mathrm{n}=3$ per group); groups 1 and 4; scaffold only as control (control group), groups 2 and 5; hDFC $3 \mathrm{D}$ culture with GM (GM group), groups 3 and 6;
hDFC 3D culture with OIM (OIM group).

All animals were anesthetized with intraperitoneal injection of $25 \mathrm{mg} / \mathrm{kg}$ pentobarbital sodium (Somnopentyl ${ }^{\circledR}$, Kyoritsu Seiyaku, Tokyo, Japan), and the surgical site was aseptically prepared. Transplantation of the 3D culture within a PTFE tube [the dimensions; inner diameter of $3 \mathrm{~mm}$, outer diameter of 4 $\mathrm{mm}$, and height of $2 \mathrm{~mm}$ ] into rats was performed as described ${ }^{12)}$. A linear skin incision was made along the edge of the skull with a \#15 surgical blade. The periosteum of the calvaria was carefully removed with a raspatorium after elevation of the skin-periosteum flap. Scaffolds or 3D cultures were inserted into a PTFE tube and placed on the calvarial surface (Fig. 1B). The overlying tissue was sutured closed (Fig. 1C).

All animals were maintained and used in accordance with the guidelines of Nihon University Intramural Animal Use (Ethics Committee Registration No: AP10MD001).

\section{Histological analysis}

Rats in groups 1-3 were sacrificed 28 days after surgery. Rats in groups 4-6 were sacrificed 56 days after surgery. The calvarial bone area including the PTFE tube was extirpated en bloc. The excised samples were fixed in $10 \%$ neutral-buffered formalin solution, decalcified in 10\% EDTA (pH 7.0), and embedded in paraffin. Semi-serial sections ( $4 \mu \mathrm{m}$ thick) were obtained in a sagittal orientation. The sections were stained with hematoxylin and eosin (H-E) and observed with light microscopy.

\section{Immunohistochemical analysis}

The sections of calvarial bone area including the PTFE tube from all groups were immunohistochemically stained. The sections were deparaffinized, and endogenous peroxidase activity was quenched by incubating in $3 \% \mathrm{H}_{2} \mathrm{O}_{2}$ in methanol for $15 \mathrm{~min}$ at room temperature. The sections were washed with PBS and incubated with mouse monoclonal antibody against rat BMP-2 (1:500; ab6285; abcam, Tokyo, Japan), rabbit polyclonal antibody against rat Runx2 (1:500; ab23981; abcam, Tokyo, Japan) or mouse monoclonal antibody against rat Osterix (1:500; ab22552; abcam, Tokyo, Japan) for $1 \mathrm{~h}$ at room temperature. Secondary antibody staining was done with reagents from ChemMate ENVISION kit/HRP (DAB; DAKO, Tokyo, Japan), according to the manufacturer's protocol. Immune complexes were visualized using 3'3'-dianibobenzidine tetradbride (DAB; Merck, Darmstat, Germany). Immunostained sections were then counterstained with Mayer's hematoxylin.

\section{Micro-CT analysis of transplants}

Quantitative image analysis of new bone formation was performed using an in vivo micro-CT system (Rigaku-mCT, Tokyo, Japan). The rats were deeply anesthetized with intraperitoneal injection of sodium pentobarbital $(25 \mathrm{mg} / \mathrm{kg})$ and individually 
Satoshi Iwai et al.: Osteogenesis of Dental Follicle Cells
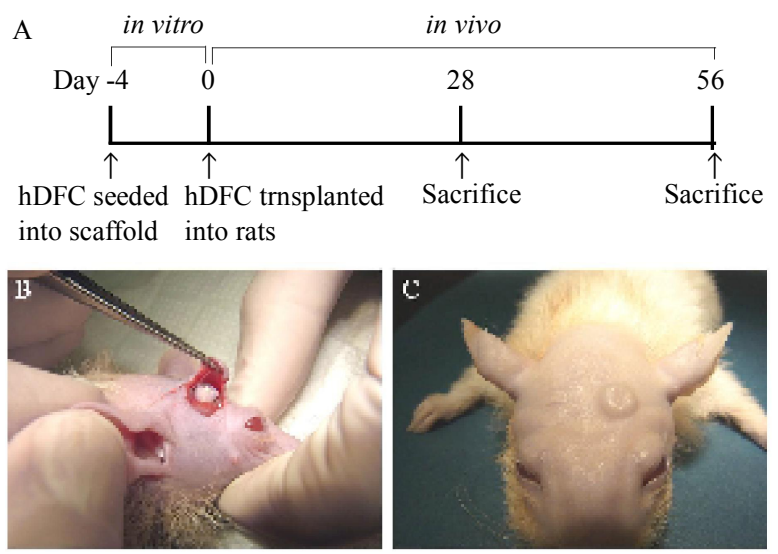

Figure 1. The procedure for evaluating bone formation by hDFC (A). hDFC cultured on a scaffold were inserted into a PTFE tube and transplanted onto the calvarial bone (B). Post-operative picture. The cutaneo-periosteal flap was closed after placement of the PTFE tube (C).

placed on the object stage. The calvarial bone at the site of the PTFE tube was scanned using a micro-CT system with an X-ray source of $90 \mathrm{kV} / 88 \mu \mathrm{A} 28$ and 56 days after surgery. Imaging of newly formed bone in the area containing the inner cavity of the PTFE tube was then performed over a full $360^{\circ}$ rotation, with an exposure time of 17 seconds. An isotropic resolution of $20 \times 20 \times$ $20 \mu \mathrm{m}$ voxels was selected, which displayed the microstructure of new bone formation of the rats. The original 3D images were displayed and analyzed with I-View software (J. Morita, Kyoto, Japan).

Analysis of new bone formation was performed in the area containing the inner cavity of the PTFE tube. The linearity of the micro-CT scanner was established by scanning a phantom containing several densities of a standard calibration material. The
3D image of newly formed bone was measured, and the structural indices of the newly formed bone were calculated using a morphometric program (TRI/3D-BON; Ratoc System Engineering, Tokyo, Japan).

The 3D analysis directly measured total volume $\left(\mathrm{TV} ; \mathrm{cm}^{3}\right)$, bone volume $\left(\mathrm{BV} ; \mathrm{cm}^{3}\right)$, and bone mineral content $(\mathrm{BMC} ; \mathrm{mg})$ of the new bone formation in the inner cavity of the PTFE tube. Volumetric density (BV/TV; \%) and bone mineral density (BMD $=\mathrm{BMC} / \mathrm{BV} ; \mathrm{mg} / \mathrm{cm} 3)$ were calculated. The bone volume to total volume ratio $(\mathrm{BV} / \mathrm{TV})$ evaluated the quantity of new bone, and the density of new bone was calculated as the BMD.

\section{Statistical analysis}

All results were expressed as the means \pm standard deviation $($ mean $\pm \mathrm{SD})$. All values were statistically analyzed with a twoway analysis of variance, and independent group variables were compared using the Mann-Whitney U-test with Bonferroni correction. Differences were considered to be significant at $P<$ 0.05 .

\section{Results \\ SEM observation of $3 D$ cultures}

SEM imaging was done to show the morphology of the $\beta$ TCP strengthening-type gelatin sponge scaffold with or without hDFC cultures after 1 and 4 days in vitro. A few spheres of various sizes $(1-20 \mu \mathrm{m})$ were seen with a smooth surface in the scaffold control culture at 1 day (Fig. 2A). Scaffolds containing hDFC cultured in GM or OIM contained many spherical nodules that were uniformly distributed throughout the scaffold after 1 day (Fig. 2B, C). The control showed few changes from day 1 to day 4 (Fig. 2D). Conversely, an increase in the number of nodules
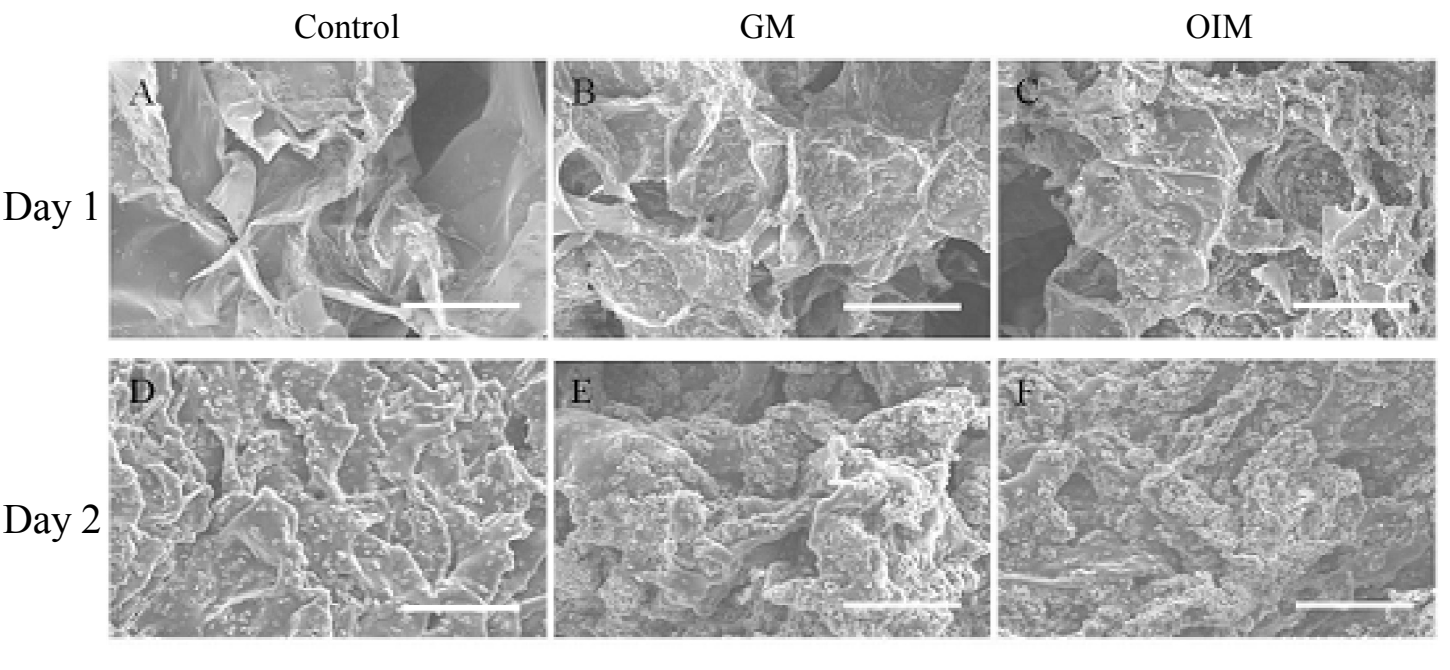

Figure 2. SEM imaging of hDFC cultivated on scaffolds $(\times 500)$. Control group (A, D), GM group $(\mathrm{B}, \mathrm{E})$, and OIM group $(\mathrm{C}, \mathrm{F})$ at 1 (A-C) and 4 days (D-F). Bars $=60 \mu \mathrm{m}$ 

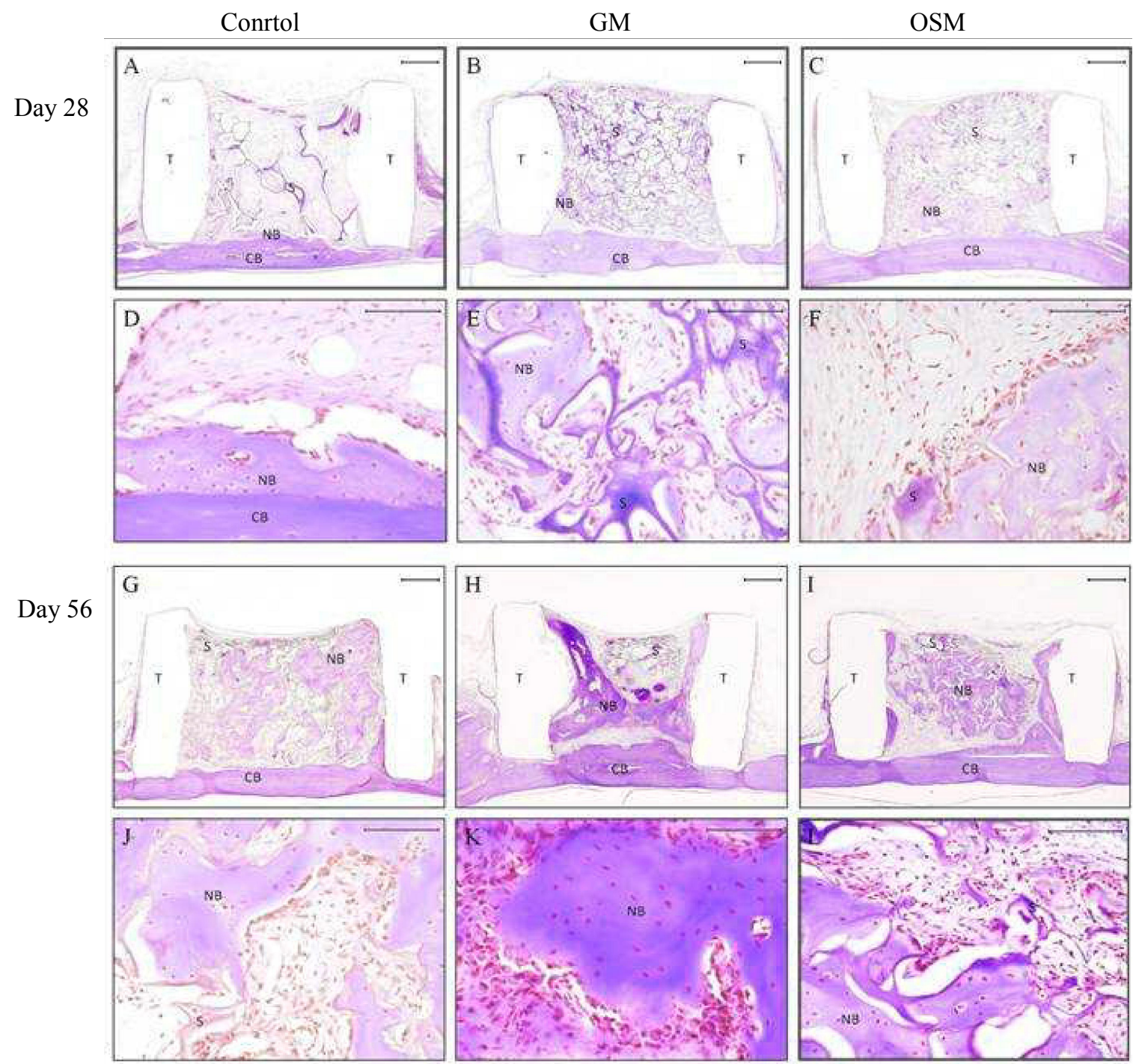

Figure 3. Histological findings with H-E staining. New bone formation was observed at 28 and 56 days (A-C, G-I, respectively). Higher magnification images of A-C and G-I are shown in D-F and J-L, respectively.

$(\mathrm{A}-\mathrm{C}, \mathrm{G}-\mathrm{I}):$ Bars $=500 \mu \mathrm{m},(\mathrm{D}-\mathrm{F}, \mathrm{J}-\mathrm{L}):$ Bars $=100 \mu \mathrm{m}(\mathrm{CB}$ : calvarial bone; T: tube; S: scaffold; NB: new bone; black arrows: osteoblast)

was observed within the depths of the scaffolds that were cultured with hDFC after 4 days (Fig. 2E, F).

\section{Histological findings}

hDFC 3D cultures were placed into PTFE tubes and transplanted between the periosteum and calvaria. Newly formed bone was seen between the cortical bone and periosteum with $\mathrm{H}$ E staining in all groups 28 and 56 days after transplantation (Fig. 3 ). The early stage of newly formed bone was observed in the scaffold in each group after 28 days (Fig. 3A-F). In the control group, thin compact bone was continuously formed with the cortical bone (Fig. 3A). Newly woven osteoids were not seen among the scaffold layers. The osteoblasts were arranged regularly along the edge of the new bone (Fig. 3D). In the GM group, a few woven osteoids were seen between scaffold layers (Fig.3B). The connective tissue in the GM group was observed enlarged capillary vessels and many spindle shaped cells with high cellular density, similar to that in the OIM group (Fig. 3E). Many woven osteoids were observed among scaffold layers in the OIM group (Fig. 3C). Enlarged capillary vessels and many spindle-shaped cells with higher cellular density were seen in the edematous connective tissue. Osteoblasts were seen around the osteoids (Fig. 3F).

More eosinophilic newly formed bone in the scaffold was observed in each group after 56 days (Fig. 3G-L). The control group showed new aggregated bone tissue in a large portion of the inner space of the PTFE tube. H-E staining was weak compared with other two groups. Woven osteoids and aggregated new bone were formed among the scaffold layers (Fig. 3G). Many osteoblasts were regularly arranged along the edge of the newly formed bone (Fig. 3J). The GM group showed new compact lamellar bone along 
Satoshi Iwai et al.: Osteogenesis of Dental Follicle Cells
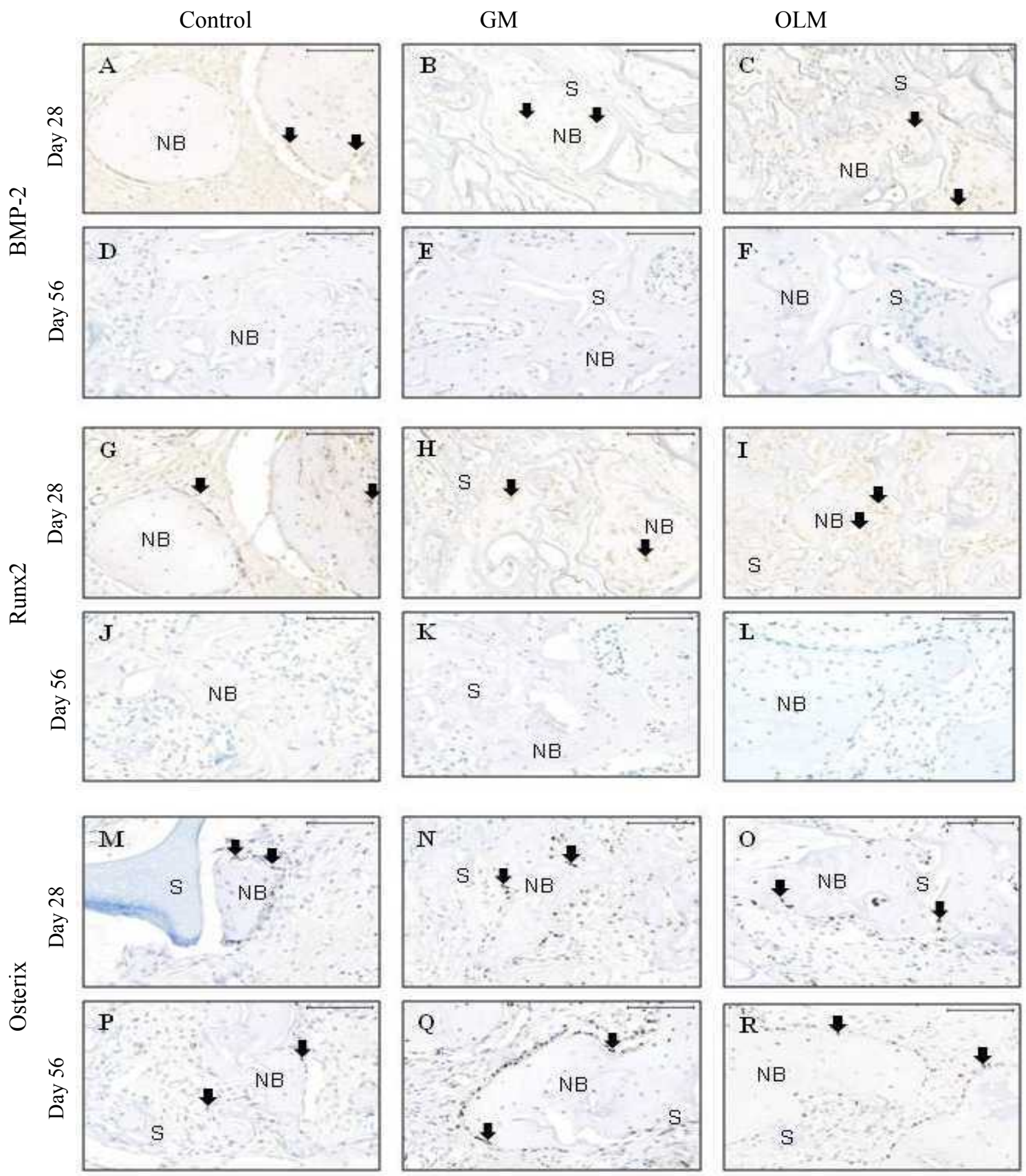

Figure 4. Representative immunohistochemistry for BMP-2, Runx2, and Osterix is shown in A-F, G-L, and M-R, respectively $(\times 400)$. Arrows indicate positive reaction.

Bars $=100 \mu \mathrm{m}(\mathrm{CB}$ : calvarial bone; T: tube; S: scaffold; NB: new bone)

the inner wall of the PTFE tube and continuous with the cortical bone (Fig. 3H). Many aggregated areas of new bone with regularly arranged osteoblasts on the edge were seen in the GM group (Fig. $3 \mathrm{~K})$. A large portion of the inner space of the PTFE tube was occupied by new aggregated bone tissue in the OIM group (Fig. 3I). Newly formed bone accompanied by bone cavities containing osteocytes was seen, and a dense arrangement of osteoblasts was seen near part of the bone edge. Moreover, osteoids were observed near the scaffold layers (Fig. 3L).

\section{Immunohistochemical findings}

Immunohistochemical findings for BMP-2, Runx2, and Osterix are shown in Figure 4A-F, G-L, and M-R, respectively. In all experimental groups, BMP-2 was observed in many spindleshaped cells scattered in the connective tissue and in osteoblasts arranged at the margin of the newly formed bone after 28 days (Fig. 4A-C). In contrast, BMP-2 was mostly negative in all groups after 56 days, although a slight positive reaction was seen (Fig. 4D-F). 

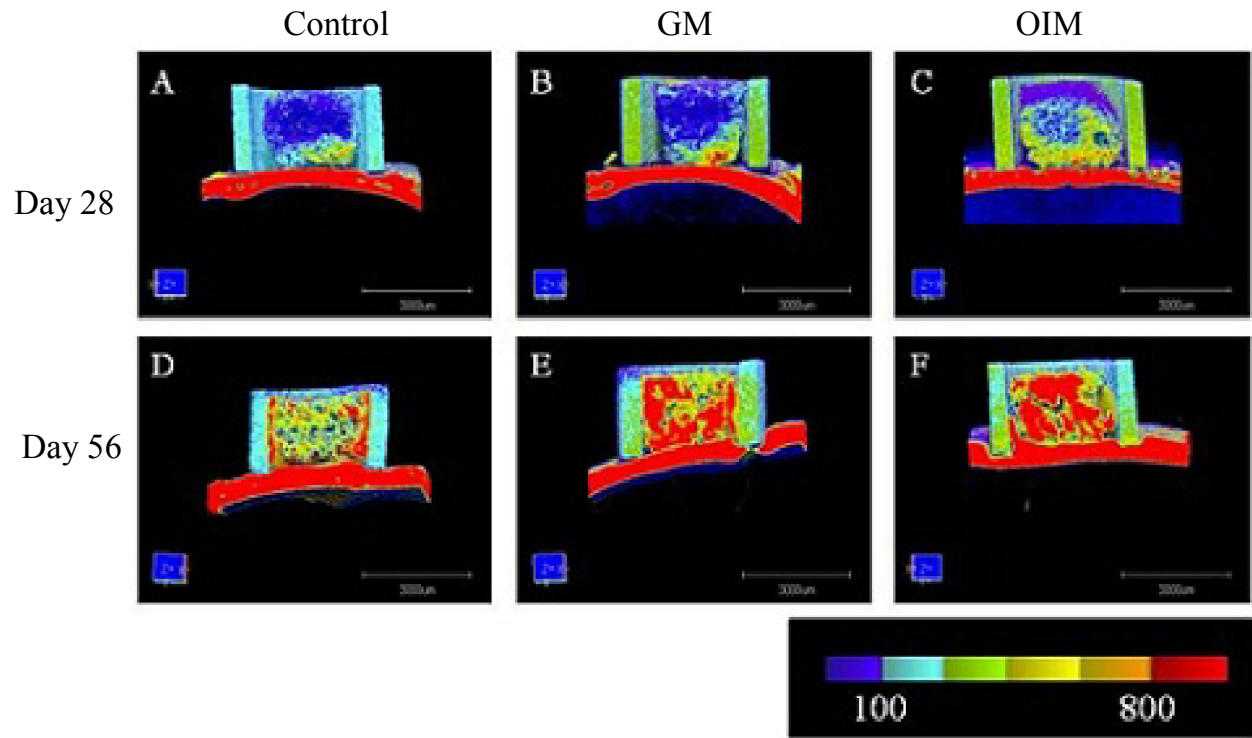

Figure 5. Color images showing bone mineral density. The inner side of the PTFE tube showed a small amount of newly formed bone at 28 days (A-C). The area of newly formed bone progressed into the PTFE tube at 56 days (D-F). The images show the control (A, D), GM (B, E), and OIM (C, F) groups. Bars $=3000 \mu \mathrm{m}$
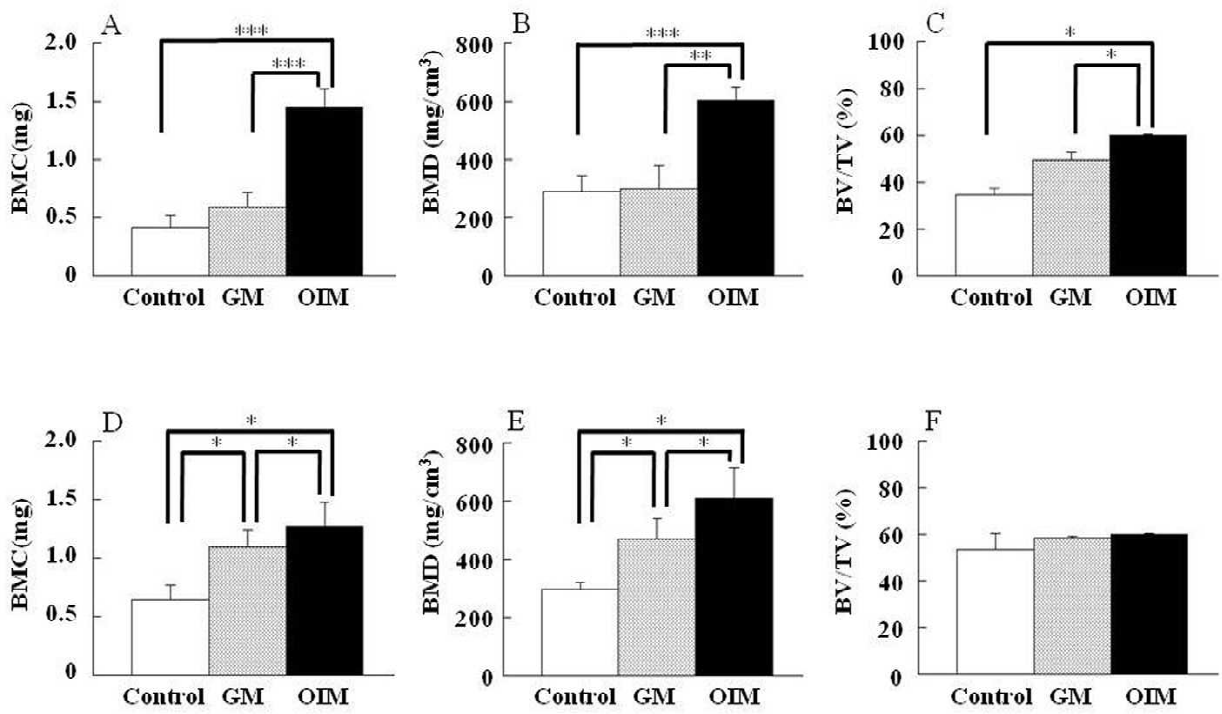

Figure 6. Quantitative measurement of BMC, BMD, and BV/TV. The columns represent the means $\pm \mathrm{SD}(*: P<0.05$, **: $\left.P<0.01,{ }^{* * *}: P<0.005\right)$. BMC (A), BMD (B), and BV/TV (C) at 28 days, and BMC (D), BMD (E), and BV/TV (F) at 56 days.

After 28 days, Runx2 was expressed in osteoblasts arranged on the margin, and also expressed osteocytes inside the newly formed bone (Fig. 4G-I). Many swollen spindle-shaped cells in the connective tissue were positive for Runx2 in both the GM and OIM hDFC cultures (Fig. 4H, I). Runx2 staining was mostly negative in all experimental groups after 56 days (Fig. 4J-L).

Positive Osterix staining was detected in a small number of osteoblasts around the newly formed bone in the control group (Fig. 4M). Strong positive Osterix staining surrounded the connective tissue in the GM and OIM groups after 28 days (Fig.
$4 \mathrm{~N}, \mathrm{O})$. Only slight staining of osteoblasts was seen in the control group after 56 days (Fig. 4P), but scattered Osterix-positive cells were seen around the newly formed bone in the GM and OIM groups (Fig. 4Q, R).

\section{Micro-CT analysis of transplants}

Micro-CT was used to quantify the mineralization of the scaffolds within the PTFE tubes at 28 and 56 days after transplantation, allowing assessment of the entire volume of the new bone. Figure 5 shows BMD color images of the transplants 
Satoshi Iwai et al.: Osteogenesis of Dental Follicle Cells

with micro-CT analysis. The inner PTFE tube contained a small amount of newly formed bone on the calvaria side after 28 days (Fig. 5A-C).

Inner PTFE tube area of newly formed bone was not observed in the scaffold-only control (Fig. 5A). A small volume of newly formed bone was seen in the GM culture (Fig. 5B), and the most newly formed bone was seen in the OIM culture (Fig. 5C). The newly formed bone area increased inside the PTFE tube after 56 days compared to after 28 days (Fig. 5D-F).

Newly formed bone was found in scattered areas above the calvaria in the scaffold-only control (Fig. 5D). In contrast, the area of newly formed bone area was found throughout the PTFE tube in the GM and OIM hDFC culture groups (Fig. 5E, F).

We next analyzed the BMC, BMD, and BV/TV using microCT (Fig. 6). The BMC value of the OIM group was significantly higher than that of the GM group and control after 28 (Fig. 6A) and 56 days (Fig. 6D). Similarly, the BMC in the GM group was higher than that of the control group after 56 days (Fig. 6D). The BMD value of the OIM group was also higher than that of the GM group and control after 28 (Fig. 6B) and 56 days (Fig. 6E). Similarly, the BMD value of the GM group was higher than that of the control group after 56 days (Fig. 6E). The BV/TV ratio of the hDFC OIM group was higher than those of the GM and control groups after 28 days (Fig. 6C), although the BV/TV ratio was not different among the three experimental groups after 56 days (Fig. $6 \mathrm{~F})$.

\section{Discussion}

Previous reports have shown that DFC isolated from human and experimental animals have the potential for mineralization in vitro and in vivo ${ }^{10,11,13)}$. However, whether hDFC that were induced to undergo osteogenic differentiation in vitro have osteogenic potential in vivo compared to undifferentiated cells was unknown. The present study demonstrated the osteogenic potential of hDFC $3 \mathrm{D}$ cultures grown in GM or OIM transplanted onto rat calvaria.

We first examined the hDFC 3D cultures using a $\beta$-TCP strengthening-type gelatin sponge as a scaffold. SEM imaging showed that the hDFC appeared as spherical nodules that proliferated in the gelatin sponge after 4 days. The hDFC were present not only at the surface but also in the inner scaffold of the sponge in both the GM and OIM cultures. Because it mimics the native tissue environment, a scaffold is essential for MSC delivery in cell-based therapy for facilitating cell adherence, migration, proliferation, and differentiation. Various types of scaffolds composed of synthetic or natural biomaterials, such as gelatin, collagen, fibrin, ceramics, etc. have been tested. Gelatin has been widely employed in tissue engineering due to its bio-degradability and low antigenicity, whereas $\beta$-TCP has been mainly employed in bone tissue engineering because of its similarity to the inorganic composition of bones. The $\beta$-TCP strengthening-type gelatin sponge is useful as a scaffold because the manufacturer improved the mechanical properties in the gelatin scaffold without altering the porous structure that enhances MSC proliferation ${ }^{14)}$.

We observed the osteogenic potential of the hDFC 3D culture in vivo. Previous studies reported that alkaline phosphate activity, which is an osteoblast differentiation marker, is enhanced in $\mathrm{hDFC}$ cultured with OIM for 4 days ${ }^{10,11}$. hDFC cultured with OIM for 4 days are in the primary osteogenic differentiation stage. In our study, hDFC proliferated in the scaffold after 4 days compared to 1 day. Therefore, hDFC cultured for 4 days were selected for transplantation into rats.

The transplanted hDFC 3D cultures were examined for new bone formation at 28 and 56 days after transplantation using H-E staining and micro-CT. Histological observations showed that the hDFC $3 \mathrm{D}$ culture transplants were at an early stage of new bone formation at 28 days. Many woven osteoids formed in the scaffold, and more were seen in the OIM group compared to the GM group and control. Micro-CT analysis showed that the BV/TV value, indicating bone volume, and BMC and BMD values, indicating the bone quality, were significantly higher in the OIM group than in the GM and control groups. Thus, new bone formation was most significantly increased in the OIM group compared to the GM and control groups at 28 days. In contrast, H-E staining showed new bone formation inside the tube in the OIM and GM groups. The BV/TV value obtained with micro-CT analysis also showed that the area of new bone was not different at 56 days among the three groups. The bone quality of the GM group was significantly increased compared to the control group as determined with BMC and BMD obtained with micro-CT analysis at 56 days.

Previous studies have demonstrated that due to preformation of calcified matrix, inducing in vitro osteogenesis in cells prior to in vivo transplantation is advantageous for rapid bone formation following subcutaneous MSC transplantation into mice ${ }^{15)}$ and post-lateral spinal fusion experiments in rabbits ${ }^{16}$. However, those reports showed that undifferentiated cells have the potential for osteogenic differentiation after in vivo transplantation, although ossification is late. Therefore, in vitro osteogenic-differentiated MSC containing hDFC may be important for successful graft transplantation in the early stage.

On the other hand, the in vivo osteogenic potential of scaffold/ MSC transplanted beneath the periosteum was observed in two different experiments, one in which MSC were pre-seeded and cultured in a scaffold using OIM, and the other in which a monolayer of MSC cultured with OIM was injected into the scaffold ${ }^{17)}$. The results indicated that pre-seeding MSC in a scaffold led to a higher osteogenic potential than the monolayer culture. These results suggested that transplanting a composite of the scaffold and cells that have undergone osteoinduction is optimal. 
Immunohistological observations revealed that BMP-2, Runx2, and Osterix, which are important factors in bone formation, were expressed in many spindle-shaped cells, which were swollen mesenchymal cells scattered in the connective tissue of new bone. BMP-2 and Runx 2 were detected in the transplants at 28 days; Osterix was detected both at 28 and 56 days. BMP-2 upregulates Runx2 expression during osteoblast differentiation ${ }^{18-20)}$. Additionally, Osterix acts downstream of Runx2 during differentiate pluripotent mesenchymal cells to the osteoblastic lineage ${ }^{21,22)}$. Thus, our immunohistochemical data reflected the BMP-2/Runx2/Osterix signaling pathway of osteoblast differentiation and new bone formation.

In this experimental model, we inserted in vitro-grown $\mathrm{hDFC}$ 3D cultures into a PTFE tube and transplanted the tube between the periosteum and calvaria. Several methods have been used to demonstrate the osteogenic potential of MSC in vivo including transplantation into a calvarial bone defect ${ }^{23-27)}$ or subcutaneous spaces ${ }^{17,28-30)}$. The method we used was useful for examining osteogenic potential because the cells were transplanted into an environment containing bone tissue in vivo without a complicated procedure such as generating a bone defect or fracture.

In conclusion, micro-CT analysis and histological observation demonstrated that new bone formation was enhanced by transplantation of human dental follicle cells. The findings suggest that hDFC will be useful as a new source of cells for bone regenerative therapy, and osteogenic-induced hDFC 3D cultures placed in a scaffold will be advantageous for new bone formation.

\section{Acknowledgments}

We would like to thank Mr. Kunihiro Suzuki (Department of Biology, Nihon University School of Dentistry at Matsudo) for his valuable advice and helpful discussion. We also wish to acknowledge Mr. Takashi Matsumoto (Diagnostic Pathology, Nihon University School of Dentistry at Matsudo) for his invaluable assistance with the immunohistochemistry technology.

\section{References}

1. Morsczeck C, Götz W, Schierholz J, Zeilhofer F, Kühn U, Möhl C, Sippel C, Hoffmann KH. Isolation of precursor cells (PCs) from human dental follicle of wisdom teeth. Matrix Biol 24:155-165, 2005

2. Yao S, Pan F, Prpic V, Wise GE. Differentiation of stem cells in the dental follicle. J Dent Res 87:767-771, 2008

3. Locke M, Feisst V, Dunbar PR. Concise review: human adipose-derived stem cells: separating promise from clinical need. Stem Cells 29:404-411, 2011

4. Schubert T, Xhema D, Vériter S, Schubert M, Behets C, Delloye C, Gianello P, Dufrane D. The enhanced performance of bone allografts using osteogenic-differentiated adiposederived mesenchymal stem cells. Biomaterials 32:8880-
8891,2011

5. Yamada Y, Ito K, Nakamura S, Ueda M, Nagasaka T. Promising cell-based therapy for bone regeneration using stem cells from deciduous teeth, dental pulp, and bone marrow. Cell Transplant 20:1003-1013, 2011

6. Chen B, Sun HH, Wang HG, Kong H, Chen FM, Yu Q. The effects of human platelet lysate on dental pulp stem cells derived from impacted human third molars. Biomaterials 33:5023-5035, 2012

7. Ten Cate AR. The development of the periodontium -a largely ectomesenchymally derived unit. Periodontol 2000 13:9-19, 1997

8. Handa K, Saito M, Yamauchi M, Kiyono T, Sato S, Teranaka T, Sampath Narayanan A. Cementum matrix formation in vivo by cultured dental follicle cells. Bone 31:606-611, 2002

9. Völlner F, Ernst W, Driemel O, Morsczeck C. A two-step strategy for neuronal differentiation in vitro of human dental follicle cells. Differentiation 77:433-441, 2009

10. Aonuma H, Ogura N, Kamino Y, Ito K, Kondoh T. Microarray analysis of human dental follicle cells in osteogenic differentiation -Gene expression of IGF-II and IGFBP-2 during osteogenic differentiation. J Hard Tissue Biol 18:2734, 2009

11. Aonuma H, Ogura N, Takahashi K, Fujimoto Y, Iwai S, Hashimoto H, Ito K, Kamino Y, Kondoh T. Characteristics and osteogenic differentiation of stem/progenitor cells in the human dental follicle analyzed by gene expression profiling. Cell Tissue Res [Epub ahead of print], 2012

12. Takiguchi S, Kuboyama N, Kuyama K, Yamamoto H, Kondoh T. Experimental study of bone formation ability with the periosteum on rat calvaria. J Hard Tissue Biol 18:149-160, 2009

13. Honda MJ, Imaizumi M, Suzuki H, Ohshima S, Tsuchiya S, Satomura K. Stem cells isolated from human dental follicles have osteogenic potential. Oral Surg Oral Med Oral Pathol Oral Radiol 111:700-708, 2011

14. Takahashi Y, Yamamoto M, Tabata Y. Osteogenic differentiation of mesenchymal stem cells in biodegradable sponges composed of gelatin and b-tricalcium phosphate. Biomaterials 26:3587-3596, 2005

15. Cheng X, Yang T, Meng W, Liu H, Zhang T, Shi R. Overexpression of GDF5 through an adenovirus vector stimulates osteogenesis of human mesenchymal stem cells in vitro and in vivo. Cells Tissues Organs 196:56-67, 2012

16. Nakajima T, Iizuka H, Tsutsumi S, Kayakabe M, Takagishi $\mathrm{K}$. Evaluation of posterolateral spinal fusion using mesenchymal stem cells: differences with or without osteogenic differentiation. Spine 32:2432-2436, 2007

17. Baba S, Inoue T, Hashimoto Y, Kimura D, Ueda M, Sakai K, Matsumoto N, Hiwa C, Adachi T, Hojo M. Effectiveness of 
Satoshi Iwai et al.: Osteogenesis of Dental Follicle Cells

scaffolds with pre-seeded mesenchymal stem cells in bone regeneration -assessment of osteogenic ability of scaffolds implanted under the periosteum of the cranial bone of rats. J Dent Mater 29:673-681, 2010

18. Lee KS, Kim HJ, Li QL, Chi XZ, Ueta C, Komori T, Wozney JM, Kim EG, Choi JY, Ryoo HM, Bae SC. Runx2 is a common target of transforming growth factor $\mathrm{b} 1$ and bone morphogenetic protein 2, and cooperation between Runx2 and Smad5 induces osteoblast-specific gene expression in the pluripotent mesenchymal precursor cell line $\mathrm{C} 2 \mathrm{C} 12$. Mol Cell Biol 20:8783-8792, 2000

19. Hanai J, Chen LF, Kanno T, Ohtani-Fujita N, Kim WY, Guo WH, Imamura T, Ishidou Y, Fukuchi M, Shi MJ, Stavnezer J, Kawabata M, Miyazono K, Ito Y. Interaction and functional cooperation of PEBP2/CBF with Smads. Synergistic induction of the immunoglobulin germline $\mathrm{Ca}$ promoter. J Biol Chem 274:31577-31582, 1999

20. Nishimura R, Hata K, Harris SE, Ikeda F, Yoneda T. Corebiding factor $\mathrm{a}_{1}$ (Cbfa1) induces osteoblastic differentiation of $\mathrm{C} 2 \mathrm{C} 12$ cells without interactions with Smad1 and Smad5. Bone 31:303-312, 2002

21. Komori T. Regulation of skeletal development by the Runx family of transcription factors. J Cell Biochem 95:445-453, 2005

22. Nakashima K, Zhou X, Kunkel G, Zhang Z, Deng JM, Behringer RR, de Crombrugghe $\mathrm{B}$. The novel zinc fingercontaining transcription factor Osterix is required for osteoblast differentiation and bone formation. Cell 108:1729, 2002

23. Xu L, Lv K, Zhang W, Zhang X, Jiang X, Zhang F. The healing of critical-size calvarial bone defects in rat with
rhPDGF-BB, BMSCs, and $\beta$-TCP scaffolds. J Mater Sci Mater Med 23:1073-1084, 2012

24. Yang Y, Hallgrimsson B, Putnins EE. Craniofacial defect regeneration using engineered bone marrow mesenchymal stromal cells. J Biomed Mater Res 99:74-85, 2011

25. Stephan SJ, Tholpady SS, Gross B, Petrie-Aronin CE, Botchway EA, Nair LS, Ogle RC, Park SS. Injectable tissueengineered bone repair of a rat calvarial defect. Laryngoscope 120:895-901, 2010

26. Schantz JT, Woodruff MA, Lam CXF, Lim TC, Machens HG, Teoh SH, Hutmacher DW. Differentiation potential of mese nchymal progenitor cells following transplantation into calvarial defects. J Mech Behav Biomed Mater 11:132-142, 2012

27. Ben-David D, Kizhner TA, Kohler T, Müller R, Livne E, Srouji S. Cell-scaffold transplant of hydrogel seeded with rat bone marrow progenitors for bone regeneration. J Craniomaxillofac Surg 39:364-371, 2011

28. Park BW, Kang EJ, Byun JH, Son MG, Kim HJ, Hah YS, Kim TH, Mohana Kumar B, Ock SA, Rho GJ. In vitro and in vivo osteogenesis of human mesenchymal stem cells derived from skin, bone marrow and dental follicle tissues. Differentiation 83:249-259, 2012

29. Agata H, Asahina I, Yamazaki Y, Uchida M, Shinohara Y, Honda MJ, Kagami H, Ueda M. Effective bone engineering with periosteum-derived cells. J Dent Res 86:79-83, 2007

30. Martin I, Muraglia A, Campanile G, Cancedda R, Quarto R. Fibroblast growth factor-2 supports ex vivo expansion and maintenance of osteogenic precursors from human bone marrow. Endocrinology 138:4456-4462, 1997 
J.Hard Tissue Biology Vol. 22(1):95-104, 2013 